\title{
Bioacumulación de hidrocarburos aromáticos policíclicos en Anadara tuberculosa (Sowerby, 1833) (Arcoida: Arcidae)
}

\section{Bioaccumulation of polycyclic aromatic hydrocarbons in Anadara tuberculosa (Sowerby, 1833) (Arcoida: Arcidae)}

\author{
Mónica Zambrano ${ }^{1 *}$, Robinson Casanova ${ }^{1}$, Jorge Prada ${ }^{1}$, Gustavo Arencibia ${ }^{2}$, Alexis Vidal ${ }^{3}$ \& Norberto \\ CAPETILLO ${ }^{2}$
}

${ }^{1}$ Grupo de Protección del Medio Marino. Centro de Investigaciones Oceanográficas e Hidrográficas del Pacífico CCCP. Vía "El Morro", Capitanía de Puerto. Tumaco, Colombia.

${ }^{2}$ Centro de Investigaciones Pesqueras. Ave. $5^{\text {ta }}$ y Cl. 248. Sta. Fé. La Habana, Cuba.

${ }^{3}$ Facultad de Biología, Universidad de La Habana. Cl. 25 \# 455 e/J El Vedado - La Habana Cuba.

*E-mail: monyzambrano@yahoo.com

\begin{abstract}
RESUMEN
Se realizaron tres pruebas de bioacumulación exponiendo moluscos bivalvos de la especie Anadara tuberculosa a concentraciones de naftaleno $(100,00 \mathrm{ppb})$, fluoranteno $(100,00 \mathrm{ppb})$ y de una mezcla de los 16 hidrocarburos aromáticos policíclicos (HAP) (15,48 ppb) considerados contaminantes prioritarios por la Environmental Protection Agency (US EPA). La menor incorporación se registró en el grupo de organismos expuestos al naftaleno con 3,40 ppb, nivel inferior al registrado en organismos expuestos a la misma concentración de fluoranteno que alcanzaron 101,13 x $10^{4}$ ppb y a 4,80 x $10^{3}$ ppb incorporados en el grupo expuesto a una muy inferior concentración de la mezcla de HAP.
\end{abstract}

Palabras Clave: Anadara tuberculosa, Bioacumulación, Hidrocarburos Aromáticos Policíclicos (HAP).

\begin{abstract}
Three bioaccumulation tests were conducted. Bivalve mollusks of the species Anadara tuberculosa were exposed to naphthalene concentrations of $100,00 \mathrm{ppb}$. They were also exposed to fluoranthene $(100,00 \mathrm{ppb})$ and a mixture of 16 polycyclic aromatic hydrocarbons (PAH); (15,48 ppb) which are considered priority pollutants by the Environmental Protection Agency (U.S. EPA). The minimum incorporation was recorded in organisms exposed to naphthalene $(3,40 \mathrm{ppb})$, a level lower than in organisms exposed to the same concentration of fluoranthene and concentration of the mixture of PAH which reached 101,13 x $10^{4} \mathrm{ppb}$ and $4,80 \mathrm{ppb} \times 10^{3}$ respectively.
\end{abstract}

KeYwords: Anadara tuberculosa, Bioaccumulation, Polycyclic Aromatic Hydrocarbons (PAHs).

\section{INTRODUCCIÓN}

Los contaminantes ambientales se acumulan en los invertebrados marinos, peces y mariscos y amenazan la salud humana, ya sea directa o indirectamente a través de la cadena alimentaria (Dixon et al. 2002). Dentro de este grupo de contaminantes se encuentran el petróleo y sus derivados, que constituyen una de las fuentes de introducción de hidrocarburos al ambiente, al ser componentes del mismo y contener la fracción más tóxica, la cual agrupa a los hidrocarburos aromáticos policíclicos (HAP) (Nair et al. 2008).
Los HAP pueden afectar significativamente a los ecosistemas, influenciando las comunidades marinas planctónicas y bentónicas, así como organismos superiores como peces y mamíferos (Pane et al. 2005); son reconocidos como potenciales carcinógenos, mutágenos y teratógenos para los seres vivos (Boscolo et al. 2007), lo que hace importante el desarrollo de estudios que evalúen su comportamiento en el ambiente.

Para realizar estudios de seguimiento a este tipo de contaminantes se ha recurrido al uso de bivalvos como organismos biomonitores (Page et al. 2005; Chęć et al. 2008), pues a través del proceso de filtración se favorece 
la bioacumulación de los diferentes xenobióticos presentes en el ambiente, siendo importantes en evaluaciones de las condiciones del medio que habitan.

En algunos países del Pacífico americano la especie Anadara tuberculosa (Sowerby 1883) ha sido empleada como organismo biomonitor en diversos estudios de contaminación marina (De la Cruz 1994; Lauestein 1995; Beliaeff et al. 1997; Herrero et al. 1999), siendo utilizada para predecir exposición, efectos y susceptibilidad ante la presencia de sustancias antropogénicas (Sericano et al. 1995). Esta especie ha sido seleccionada en monitoreos tendientes a evaluar contaminantes como metales pesados, plaguicidas e hidrocarburos en el Pacífico colombiano (Marrugo 1993; Casanova 1995; Sericano et al. 1995; Calero \& Zambrano 1997) donde además se destaca como el molusco de mayor interés comercial, llegando a constituir el 96\% de la producción total de bivalvos del país (INCODER, 2007).

Teniendo en cuenta los diversos eventos de contaminación por petróleo registrados en la zona sur del Pacífico colombiano y la alta demanda que tienen estos organismos como fuente de alimento para la población, se realizó un seguimiento a la bioacumulación de naftaleno, fluoranteno y de una mezcla de los 16 HAP considerados contaminantes de interés en función de sus aplicaciones industriales y como contaminantes ambientales tóxicos (IARC 1983; ATSDR 2011).

El presente estudio se desarrolló con el fin de determinar los niveles incorporados por los organismos tras la exposición a los compuestos previamente mencionados, y el comportamiento en cuanto a la retención y depuración de los mismos.

\section{MATERIALES Y MÉTODOS}

Los organismos de ensayo se recolectaron en la Bahía de Tumaco en el Pacífico colombiano (153'10" N y 78³9'50" W) y fueron adquiridos a través de pescadores artesanales, trasladados al laboratorio del Centro de Investigaciones Oceanográficas e Hidrográficas del Pacífico - CCCP en donde fueron sometidos a un periodo de aclimatación en tanques de 250L.

La duración del periodo de aclimatación para las pruebas realizadas con naftaleno y fluoranteno fue de 5 días, mientras que para la mezcla de HAP este periodo se extendió a 10 días, con el fin de que los organismos no solo se adaptaran a las condiciones de laboratorio, sino que también depuraran los niveles de compuestos de alto peso molecular (PM) que pudieran haber acumulado en el medio natural, ya que estos tienen una mayor persistencia con respecto a los de bajo y medio PM. Este periodo se determinó teniendo en cuenta que para las pruebas realizadas con sedimentos marinos se requieren dos días de depuración (APHA, AWWA,WEF, 2005).

Previo al inicio de las pruebas se evaluó la estabilidad del naftaleno y fluoranteno en el agua de disolución bajo condiciones ambientales. Cada compuesto se diluyó en acetona y luego para cada uno de ellos se preparó un recipiente de vidrio con $3 \mathrm{~L}$ de agua marina filtrada, al que se adicionaron $5 \mathrm{~mL}$ de una solución de $1,2 \times 10^{3}$ ppm, obteniendo una concentración de $2 \times 10^{3} \mathrm{ppb}$ de cada compuesto. Se tomaron $0,5 \mathrm{~L}$ de agua de cada recipiente con periodicidad de seis horas para determinar la concentración remanente a lo largo de la prueba.

Las pruebas de bioacumulación fueron de tipo semiestático, con recambios periódicos del $100 \%$ volumen de agua (20 L) cada 24 horas en los recipientes: de control (agua marina filtrada; y solución de acetona), y para los recipientes de ensayo con las tres soluciones, sin suministro de aireación. El agua de recambio se sometía previamente a varias horas de aireación con el fin de incrementar la concentración de oxígeno disuelto (OD). Diariamente se registraron los parámetros fisicoquímicos (salinidad, $\mathrm{pH}$, temperatura y OD) empleando un equipo multiparámetros WTW Multi 340i. Así mismo se proporcionó alimento a los organismos, suministrando diatomeas ( $1 \mathrm{~L}$ por recipiente) con una densidad promedio de $3 \times 10^{6}$ células $\mathrm{mL}^{-1}$.

Se emplearon acuarios de vidrio aforados a $20 \mathrm{~L}$ como recipientes de ensayo y en cada uno de ellos se expusieron un total de 10 organismos, cantidad requerida como muestra para los análisis tendientes a determinar la concentración de los contaminantes. Antes de iniciar cada bioensayo se tomó una muestra para determinar el contenido inicial del compuesto(s) en los organismos.

En las pruebas realizadas con naftaleno, y fluoranteno se expusieron 60 organismos a $100 \mathrm{ppb}$ del compuesto de ensayo por un periodo de 30 días, mientras que para la prueba realizada con la mezcla de 16 HAP se expusieron 120 organismos a 15 ppb por 45 días. Los compuestos de ensayo, sus características fisicoquímicas y de toxicidad se relacionan en la tabla 1.

La duración de los ensayos se determinó teniendo en cuenta que para pruebas de bioacumulación en campo la exposición abarca 30 días o mas para evaluar concentraciones de contaminantes en tejidos; si los compuestos son debajo PM este periodo puede ser inferior, pero para compuestos orgánicos de alto PM se puede prolongar la prueba a 60 o 90 días (APHA, AWWA, WEF, 2005). 


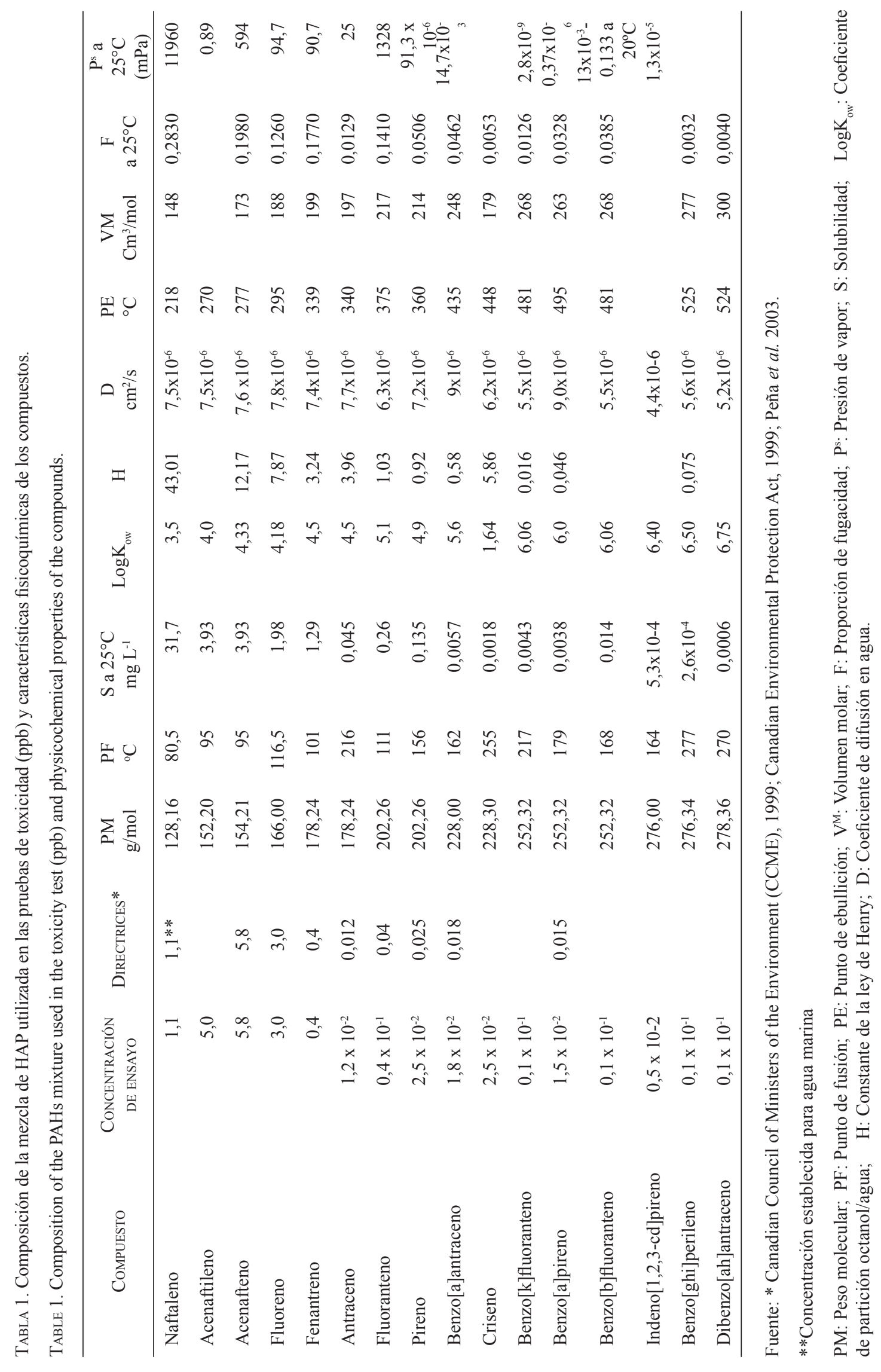


Las concentraciones empleadas en la mezcla de HAP equivalen a niveles guía de calidad del Canadian Council of Ministres of the Environment (CCME 1999), los cuales no representan riesgo para la vida acuática, pero teniendo en cuenta que las mezclas de HAP pueden tener efectos tóxicos aditivos/sinérgicos, no se incrementó la concentración de los compuestos.

Para los compuestos que no cuentan con un nivel de referencia se estableció una concentración inferior a la estipulada para el antraceno, que cuenta con la mayor restricción entre los compuestos que pertenecen a este grupo dentro de la guía. Las concentraciones de naftaleno y fluoranteno se establecieron empleando niveles superiores a los establecidos en los criterios de calidad, con el fin de proporcionar una mayor disponibilidad del compuesto en el medio.

Las soluciones de ensayo se prepararon a partir de soluciones patrón de naftaleno, y fluoranteno de 4,0 x $10^{5}$ ppb (preparadas en acetona). La mezcla de HAP requirió la preparación de tres soluciones patrón (de acuerdo a la similitud en la concentración requerida para cada compuesto) empleando acetona como solvente, las cuales fueron adicionadas en diferentes alícuotas, con el fin de obtener las concentraciones estipuladas para adelantar la prueba, obteniendo una concentración final de.15,48 ppb (de los 16 analitos) requerida en los recipientes de ensayo.

En cada prueba se emplearon dos tipos de recipientes de control, uno con agua de disolución y otro con adición del solvente (acetona), en volúmenes equivalentes al empleado en la adición del compuesto. A cada control se asignaron tres recipientes (un original con dos réplicas).

La talla media de los organismos de ensayo fue de $4,88 \mathrm{~cm}$ de longitud total (LT) y la determinación de los niveles de HAP incorporados se realizó con periodicidad de cinco días, tomando 10 organismos como muestra, se extrajo el tejido contenido en las valvas y se homogenizó, tomando del mismo una muestra de aproximadamente $10 \mathrm{~g}$ siguiendo el procedimiento descrito por Bruhn et al. (1987) para obtener las fracciones de hidrocarburos presentes en la muestra.

Con las fracciones obtenidas se realizó la cuantificación a través de un sistema de CG-EM, conformado por las siguientes unidades: inyector 7683 Agilent Technologies; cromatógrafo de gases $6890 \mathrm{~N}$ Network Agilent Technologies; detector selectivo de masas 5973 Network Agilent Technologies, automuestreador 7683 Agilent Technologies, siguiendo las metodologías descritas por Russell et al. (2002).

\section{RESULTADOS}

Durante la prueba realizada para evaluar la estabilidad del naftaleno en el medio se determinó una pérdida de aproximadamente el $50 \%$ del compuesto cada seis horas, pasando de una concentración inicial de $2 \times 10^{3} \mathrm{ppb}$ a 1,04 $\mathrm{x} 10^{3} \mathrm{ppb}$ transcurridas las primeras seis horas de prueba y a $500 \mathrm{ppb}$ transcurridas 12 horas, razón por la cual durante la prueba de bioacumulación con este compuesto se hizo una adición del $50 \%$ de la concentración inicial suministrada con periodicidad de 6 horas. La prueba correspondiente al fluoranteno no presentó disminución en la concentración inicial aun transcurridas 24 horas de ensayo.

La concentración inicial determinada en los organismos previo inicio de las pruebas fue de $10,39 \times 10^{2} \mathrm{ppb}$ para el naftaleno y de $58,80 \times 10^{2}$ ppb para el fluoranteno tras cinco días de aclimatación, y de 15,30 ppb para los 16 HAP contenidos en la mezcla luego de un periodo de aclimatación de 10 días tal y como se puede observar en la tabla 2 , en la que también se relacionan los niveles de cada compuesto determinados en los organismos a lo largo de las pruebas.

Durante el periodo de exposición al naftaleno la concentración del compuesto en los tejidos de organismos varió entre $1,57 \times 10^{3} \mathrm{ppb}$ y $3,42 \times 10^{3} \mathrm{ppb}$ correspondientes a los días 15 y 20 respectivamente con ascensos y descensos a lo largo de la prueba (figura 1); el fluoranteno presentó niveles de 194,77 x $10^{3} \mathrm{ppb}$ tras cinco días de exposición a $101,13 \times 10^{4} \mathrm{ppb}$ luego de 25 días con un comportamiento ascendente durante la mayor parte de la prueba y un descenso importante en un corto periodo de tiempo (Fig. 2), mientras que las concentraciones determinadas en organismos expuestos a la mezcla de los 16 HAP estuvieron entre $2,43, \times 10^{3} \mathrm{ppb}$ para el quinto día de exposición y $5,48 \times 10^{3} \mathrm{ppb}$ para el día 45 .

Los niveles de HAP incorporados por organismos tras la exposición a la mezcla fueron mayores para el acenafteno y fluoreno, siguiéndole en concentración el acenaftileno y fenantreno. Solo el naftaleno y el fluoranteno no fueron acumulados y el benzo[a]pireno fue el compuesto que se incorporó en menor concentración, como puede observarse en la Fig. 3.

Soloseregistrómortalidaden los organismos correspondientes a la prueba realizada con la mezcla de HAP, alcanzando un 33,33\% (entre los días 13 y 33) en los recipientes correspondientes al control y un 56,66\% (entre los días 16 y 22) en los controles con adición de acetona. La mortalidad entre los organismos expuestos a la mezcla registró un 43,5 $\%$ entre los días dos y 29 de iniciada la prueba. 


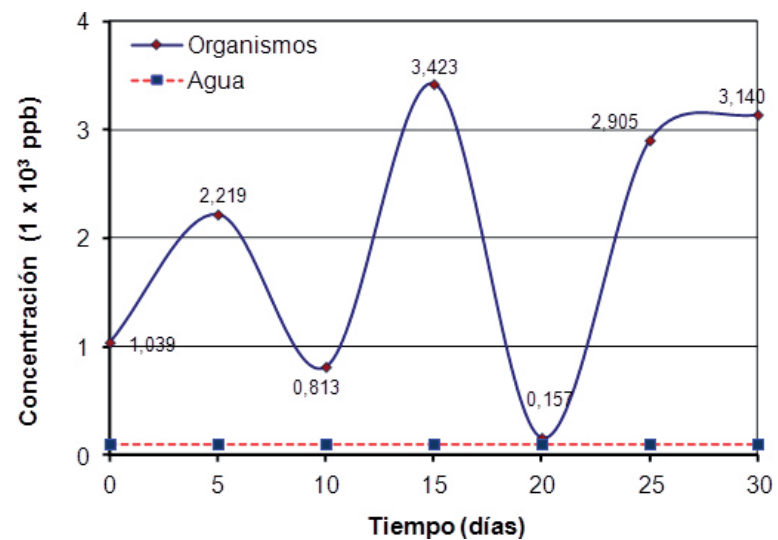

Figura 1. Comportamiento del naftaleno en Anadara tuberculosa durante la prueba de bioacumulación (ppb / peso seco)

FIGURE 1. Behavior of naphthalene in Anadara tuberculosa during the bioaccumulation test (ppb / dry weight).

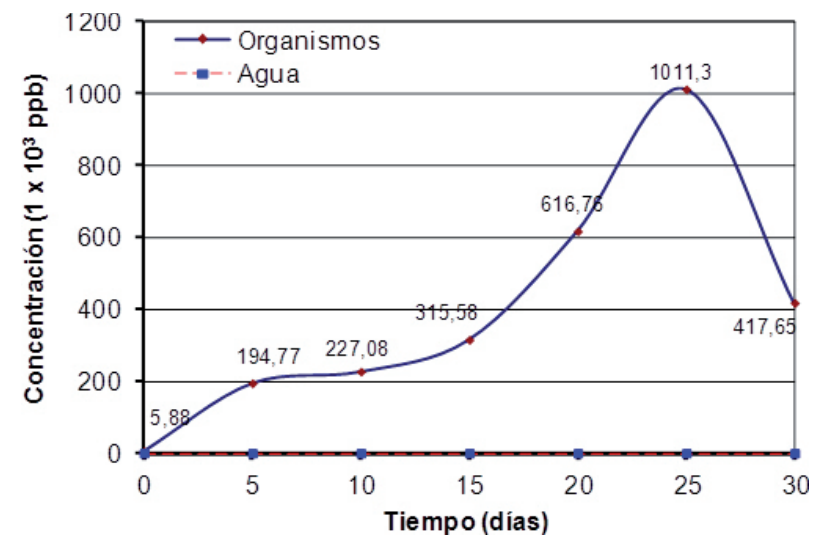

FIgURA 2. Comportamiento del fluoranteno en Anadara tuberculosa durante la prueba de bioacumulación ( $\mathrm{ppb} /$ peso seco).

FIgURE 2. Behavior of fluoranthene in Anadara tuberculosa during the bioaccumulation test (ppb / dry weight).

TABLA 2. Concentración de naftaleno, fluoranteno y HAP en Anadara tuberculosa durante las pruebas de bioacumulación (ppb/ peso seco).

TABLE 2. Concentration of naphthalene, fluoranthene and PAHs in Anadara tuberculosa during the bioaccumulation test (ppb/ dry weight)

\begin{tabular}{|c|c|c|c|c|c|c|c|c|c|c|}
\hline \multirow{2}{*}{$\begin{array}{l}\text { Tiempo (días) } \\
\text { Compuesto }\end{array}$} & \multicolumn{10}{|c|}{ CONCENTRACIÓN } \\
\hline & $\mathbf{0}$ & 5 & 10 & 15 & 20 & 25 & 30 & 35 & 40 & 45 \\
\hline Naftaleno & 1039,0 & 2219,0 & 813,0 & 3423,0 & 157,0 & 2905,0 & 3140,0 & & & \\
\hline Fluoranteno & 5880,0 & 194770,0 & 227080,0 & 315580,0 & 616760,0 & 1011300,0 & 417650,0 & & & \\
\hline \multicolumn{11}{|l|}{ Mezcla de HAP } \\
\hline Naftaleno & N.D & N.D & N.D & N.D & N.D & N.D & N.D & N.D & N.D & N.D \\
\hline Acenaftileno & 15,0 & 313,0 & 200,0 & 564,0 & 590,0 & 364,0 & 370,0 & 540,0 & 530,0 & 610,0 \\
\hline Acenafteno & 13,0 & 757,0 & 347,0 & 1236,0 & 1210,0 & 838,0 & 790,0 & 1156,0 & 1070,0 & 1290,0 \\
\hline Fluoreno & 14,0 & 440,0 & 480,0 & 575,0 & 630,0 & 506,0 & 704,0 & 637,0 & 610,0 & 730,0 \\
\hline Fenantreno & 12,0 & 320,0 & 510,0 & 360,0 & 410,0 & 350,0 & 600,0 & 440,0 & 420,0 & 520,0 \\
\hline Antraceno & 20,0 & 102,0 & 220,0 & 114,0 & 193,0 & 112,0 & 294,0 & 155,0 & 190,0 & 195,0 \\
\hline Fluoranteno & N.D & N.D & N.D & N.D & N.D & N.D & N.D & N.D & N.D & N.D \\
\hline Pireno & 25,0 & 67,0 & 185,0 & 90,0 & 130,0 & 100,0 & 165,0 & 90,0 & 150,0 & 100,0 \\
\hline Benzo $[a]$ antraceno & 1,7 & 54,0 & 100,0 & 87,0 & 160,0 & 100,0 & 148,0 & 175,0 & 98,0 & 120,0 \\
\hline Criseno & 0,5 & 102,0 & 140,0 & 161,0 & 270,0 & 173,0 & 250,0 & 332,0 & 150,0 & 210,0 \\
\hline Benzo $[k]$ fluoranteno & 1,6 & 57,0 & 110,0 & 114,0 & 163,0 & 122,0 & 172,0 & 226,0 & 100,0 & 130,0 \\
\hline Benzo $[a]$ pireno & 4,7 & 32,0 & 85,0 & 69,0 & 130,0 & 75,0 & 153,0 & 142,0 & 75,0 & 950,0 \\
\hline Benzo $[b]$ fluoranteno & 4,3 & 47,0 & 110,0 & 109,0 & 147,0 & 118,0 & 170,0 & 240,0 & 110,0 & 140,0 \\
\hline $\begin{array}{l}\text { Indeno }[1,2,3- \\
c d] \text { pireno }\end{array}$ & 19,0 & 65,0 & 100,0 & 205,0 & 250,0 & 173,0 & 308,0 & 346,0 & 145,0 & 224,0 \\
\hline Benzo[ghi]perileno & 1,7 & 40,0 & 140,0 & 120,0 & 118,0 & 110,0 & 216,0 & 155,0 & 110,0 & 130,0 \\
\hline Dibenzo $[a h]$ antraceno & 11,0 & 40,0 & 137,0 & 107,0 & 110,0 & 96,0 & 220,0 & 168,0 & 113,0 & 140,0 \\
\hline Sumatoria HAP & 143,5 & 2436,0 & 2864,0 & 3911,0 & 4511,0 & 3237,0 & 4560,0 & 4802,0 & 3871,0 & 5489,0 \\
\hline
\end{tabular}

N.D No detectado 

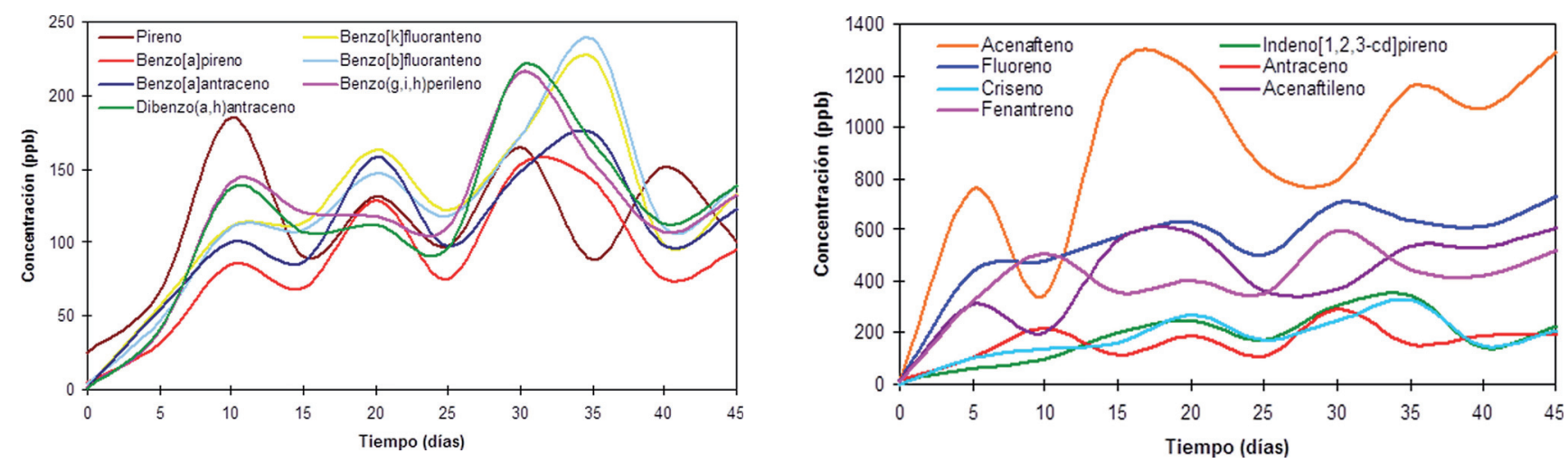

FigURA 3. Comportamiento de los HAP en Anadara tuberculosa durante la prueba de bioacumulación.

FIGURE 3. Behavior of PAH in Anadara tuberculosa during the bioaccumulation test.

TABla 3. Comportamiento de los parámetros fisicoquímicos durante las pruebas de bioacumulación.

TABLE 3. Behavior of physicochemical parameters during the bioaccumulation testing.

\begin{tabular}{lcccc}
\hline \multicolumn{1}{c}{$\begin{array}{c}\text { Parámetro } \\
\text { Compuesto }\end{array}$} & $\begin{array}{c}\text { Oxigeno Disuelto } \\
\mathrm{mg} \mathrm{O}_{2} \mathrm{~L}^{-1}\end{array}$ & $\mathrm{pH}$ & $\begin{array}{c}\text { Salinidad } \\
\% \text { o }\end{array}$ & $\begin{array}{c}\text { Temperatura } \\
{ }^{\circ} \mathrm{C}\end{array}$ \\
\hline Naftaleno & $2.97-7.64$ & $7.52-7.93$ & $30.2-31.6$ & $22.2-26.3$ \\
Fluoranteno & $3.28-7.24$ & $7.30-7.99$ & $28.1-30.9$ & $24.1-27.3$ \\
Mezcla HAP & $3.25-7.80$ & $7.91-8.24$ & $29.3-30.0$ & $23.3-25.7$ \\
\hline
\end{tabular}

Los registros correspondientes a las mediciones de algunos parámetros fisicoquímicos fueron muy similares en las tres pruebas realizadas, presentando niveles que variaron entre 2,97 y $7,80 \mathrm{mg} \mathrm{O}_{2} \mathrm{~L}^{-1}$ de OD; 7,3 y 8,24 para el $\mathrm{pH}$; 28,1 y 31,6 psu para la salinidad; y de 22,2 a $26,3{ }^{\circ} \mathrm{C}$ de temperatura (Tabla 3 ), parámetros que se encuentran dentro de los rangos vitales conocidos para la especie.

\section{DISCUSION}

La bioacumulación es el proceso por el cual ciertas sustancias tienden a acumularse en los tejidos vivos, se relaciona con las características lipofílicas, fisiológicas y bioquímicas de los organismos. Su evaluación es importante en la valoración científica de los riesgos que las sustancias químicas pueden representar para los seres humanos y el medio ambiente y es un enfoque actual de los esfuerzos de reglamentación (Arnot \& Gobas 2006).

En el caso específico de los HAP, estos compuestos son bioacumulables debido a sus características lipofílicas, por lo que una vez ingeridos su eliminación metabólica se hace lenta y dificultosa, tendiendo a acumularse en órganos de almacenamiento como por ejemplo los tejidos grasos (Fernández \& Freire 2005).

El naftaleno es un compuesto moderadamente volátil y por esa razón su concentración disminuye con mayor facilidad en el medio frente a otros compuestos del mismo grupo, característica asociada con su bajo PM que a la vez lo hace rápidamente disponible a los organismos y facilita su movilidad con respecto a otros HAP, siendo retenido por cortos periodos de tiempo. Estas características explican las fluctuaciones en cuanto a ascensos y descensos en la concentración de este compuesto presentadas por los organismos a lo largo de la prueba.

El fluoranteno es más persistente que los compuestos más ligeros del grupo y se acumula en mayor proporción con respecto a los mismos tal y como se ha reportado en evaluaciones realizadas con otras especies de moluscos 
(Hellou et al. 1993, Krishnakumar et al. 1994; 1997). Su rápido incremento en concentración frente al naftaleno se relaciona con los diferentes grados de afinidad lipofílica, toxicidad y persistencia de los HAP, que son más elevados cuanto mayor es el número de anillos de la molécula; su posterior descenso sugiere un proceso de depuración tras alcanzar un determinado nivel de incorporación, tal y como se ha observado en otros invertebrados expuestos a este compuesto (Krishnakumar et al. 1997; Landrum et al. 2002).

Durante la prueba realizada con la mezcla de HAP no se presentó bioacumulación de naftaleno ni de fluoranteno, esto obedece a que como se ha mencionado previamente, el naftaleno cuenta con una alta volatilidad y junto con el fluoranteno, la no incorporación también puede relacionarse con la baja concentración suministrada de ambos compuestos.

La bioacumulación fue mayor para los compuesto de alto PM teniendo en cuenta las proporciones registradas a partir de las concentraciones suministradas, a este comportamiento se suma una baja variabilidad a lo largo del periodo de exposición; mientras que los compuestos de menor PM presentaron un comportamiento inverso, situación derivada de la mayor disponibilidad y menor persistencia que les caracteriza frente a los compuestos de mayor PM. La variabilidad en la concentración de HAP incorporados por los organismos es un comportamiento usual ante la exposición a niveles de contaminantes en el medio, ya que estos incorporan los compuestos y a la vez pueden realizar excreción de los mismos, aumentando y disminuyendo la concentración en tejidos, aún en presencia de los compuestos en el medio (Calero \& Zambrano 1997; CSIC 2003).

En cuanto a la mortalidad registrada durante la prueba con la mezcla de HAP, esta no puede asociarse con problemas de salud de los organismos, pues durante el periodo de aclimatación (10 días) la sobrevivencia fue de un $100 \%$. Por otra parte, el que se registrara mortalidad en los organismos de ambos tipos de control (agua de disolución y aguaacetona) descarta la posibilidad de que la misma asociara exclusivamente con la exposición al solvente y/o a la mezcla suministrada.

Dado que las pruebas realizadas con naftaleno y fluoranteno no registraron mortalidad, se hace evidente la presencia de un factor adicional a la exposición al solvente y a la mezcla, el cual ejerció una influencia negativa sobre los organismos; siendo posible que exista una relación con el periodo de aclimatación, el cual fue superior al abarcado en las pruebas restantes, en las que no se registró mortalidad. De hecho, en pruebas de bioacumulación en las que organismos de esta especie fueron expuestos a una solución hidrocarburos derivados del petróleo (Calero \& Zambrano 1997), en los que los periodos de aclimatación fueron inferiores a cinco días, tampoco se registró mortalidad.

Con respecto al comportamiento de los parámetros fisicoquímicos, este fue muy similar para las tres pruebas realizadas, por lo que tampoco puede relacionarse con la tasas de mortalidad presentada en la prueba, además los niveles registrados son adecuados para ambas especies teniendo en cuenta que habitan en zonas fangosas que presentan cambios bruscos en los diferentes parámetros fisicoquímicos causados por los cambios mareales (Prahl et al. 1990)

La disminución de la mortalidad y su posterior desaparición tras un determinado periodo de tiempo, puede estar relacionada con una respuesta de adaptación por parte de los organismos tras un periodo de mayor sensibilidad, que sin duda se acentúa con la presencia del solvente y la mezcla tóxica en el medio.

\section{CONCLUSIONES}

Los altos niveles de naftaleno, fluoranteno y HAP incorporados por los organismos con respecto a la concentración proporcionada durante las pruebas, evidencian la alta capacidad bioacumulativa de la especie $A$. tuberculosa, lo que ratifica su importancia como organismo biomonitor y como una importante herramienta en la evaluación de contaminantes en ecosistemas estuarinos del Pacífico colombiano.

La elevada tasa de mortalidad presentada inicialmente durante las pruebas realizadas con la mezcla de los 16 HAP y su posterior desaparición, es una clara evidencia de la capacidad de adaptación de los organismos ante la presencia de compuestos tóxicos en el ambiente.

La capacidad de A. tuberculosa para incorporar altos niveles de contaminantes puede representar riesgo para los consumidores de estos organismos si en su hábitat se presentan ingresos frecuentes, o niveles importantes de contaminantes asociados con el petróleo y sus derivados.

\section{AGRADECIMIENTOS}

Los autores agradecen a la Dirección del Centro de Investigaciones Oceanográficas e Hidrográficas del Pacífico - CCCP y a Ecopetrol, por la financiación proporcionada para el desarrollo del presente estudio. 


\section{BIBLIOGRAFIA}

Apha, Awwa,Wef. 2005. Standard Methods for the Examination of Water and Wastewater. 21st ed. American Public Health Association. Washington, DC. Part 8000 Toxicity. 8, 94100

Arnot, J.A. \& Gobas, F.A.P.C. 2006. A review of bioconcentration factor $(\mathrm{BCF})$ and bioaccumulation factor (BAF) assessments for organic chemicals in aquatic organisms. Environ. Rev. 14 (4): 257-297.

ATSDR. 2011. Citing Electronic Resources. 2007 CERCLA Priority List of Hazardous Substances. Atlanta. URL: http:www. atsdr.cdc.gov/cercla/07list.html Accesado: Mayo 26, 2011.

Beliaeff, B., O'connor, T. P. Daskalakis, D.K. \& Smith, P.J. 1997. U.S. Mussel Watch data from 1986-1994: Temporal Trend detection at large spatial scales. Environ. Sci. Technol. 3: 1411-1415.

Boscolo, R., Cacciatore, F. \& Giovanardi, O. 2007. Polycyclic aromatic hydrocarbons (PAHs) in transplanted Manila clams (Tapes philippinarum) from the Lagoon of Venice as assessed by PAHs/shell weight index: A preliminary study. Mar. Pollut. Bull. 55, 485-493.

Bruhn, C., Duke, V., Lecaros, O., Marrugo, A. \& Sonnerholzner, S. 1987. Informe del Curso Regional CPPS/PNUMA/ COI. Técnicas Analíticas para la Determinación de Hidrocarburos del Petróleo en organismos y Sedimentos Marinos en el Pacífico Sudeste. 23 pp.

Calero, L. \& Zambrano, M. 1997. Bioacumulación de hidrocarburos aromáticos del petróleo en un molusco bivalvo Anadara tuberculosa. Bol. Cient. CCCP. 6: 131137.

Canadian Environmental Protection Act. 1999. Polycyclic Aromatic Hydrocarbons. Priority Substances List Assessment Report. Government of Canada/Environment Canada/Health Canada. 63 pp.

Casanova, R. 1995. Estudio de la contaminación marina por compuestos organoclorados en el Pacífico colombiano. Bol. Cient. CCCP . 5: 146 - 160.

CCME. 1999. Canadian Environmental Quality Guidelines. En: GARRETT, C. 2004. Priority Substances of Interest in the Georgia Basin Profiles and background information on current toxics issues. Report of the Canadian Toxics Work Group. GBAP Publication No. EC/GB/04/79.

Chęć, E., Podgórska, B. \& Węgrzyn, G. 2008. Comparison of the use of mussels and semipermeable membrane devices for monitoring and assessment of accumulation of mutagenic pollutants in marine environment in combination with a novel microbiological mutagenicity assay. Environ. Monit. Assess. 140: 83-90.

CsIC. 2003. Citing Electronic Resources. Consejo Superior de Investigaciones Científicas. URL: http://csicprestige.iim. csic.es/informes/info15.pdf Accesado: June 03, 2011.

De la Cruz, E. 1994. Stable pollutants in the bivalve Anadara tuberculosa, from the Nicoya Gulf, Costa Rica. En: Otchere, F.A. 2005. Organochlorines (PCBs and pesticides) in the bivalves Anadara (Senilis) senilis, Crassostrea tulipa and Perna perna from the lagoons of Ghana. Sci. Total. Environ. 348: 102-114

Dixon, D.R., Pruski, A.M., Dixon, L.R. \& JhA, A.N. 2002.
Marine invertebrate ecogenotoxicology: a methodological overview. Mutagenesis 17, 495-507.

Fernández, L. \& Freire, J. 2005. Relaciones entre la salud de los ecosistemas marinos y la seguridad alimentaria: La marea negra del Prestige como un caso de estudio. En Ferreras, J. \& Freire, J. (eds). Seguridad Alimentaria (I): Pesca, Acuicultura y Marisqueo. Biblioteca Monográfica de la Fundación Instituto de Estudios Políticos y Sociales, A Coruña, 91-128.

Hellou, J., Upshall, C., Payne, J.F., Naidu, S. \& Paranjape, M.A. 1993. Total unsaturated compounds and polycyclic aromatic hydrocarbons in mollusks collected from waters around Newfoundland. Arch. Environ. Contam. Toxicol. 24: 249-257.

Herrero, L., Palacios, A., Hun, L. \& Vega, F. 1999. Ausencia de detección de enterovirus en bivalvos Anadara tuberculosa (Bivalvia: Arcidae) por contaminación química en el Pacífico de Costa Rica. Rev. Biol. Trop. 47: 419-427.

IARC. 1983. Polynuclear Aromatic Compounds: Part 1, Chemical, environmental and experimental data. IARC Monographs on the Evaluation of Carcinogenic Risks to Humans. World Health Organization. 32: 95-431.

INCODER. 2007. Sistema de información sectorial pesquero. En: Lovatelli, A., Farias, A. \& Uriarte, I. (eds). Estado actual del cultivo y manejo de moluscos bivalvos y su proyección futura: factores que afectan su sustentabilidad en América Latina. Taller Técnico Regional de la FAO. 20-24 de agosto de 2007, Puerto Montt, Chile. FAO Actas de Pesca y Acuicultura. No. 12. Roma, FAO. 115-128.

Krishnakumar, P.K., Casillas, E. \& Varanasi, U. 1994. Effects of environmental contaminants on the health of Mytilus edulis from Puget Sound, Washington, USA. I. Cytochemical measures of lysosomal responses in the digestive cells using automatic image analysis. Mar. Ecol. Prog. Ser. 106: 249-261.

Krishnakumar, P.K., Casillas, E. \& Varanasi, U. 1997. Cytochemical reponses in the digestive tissue of Mytilus edulis complex exposed to microencapsulated $\mathrm{PAH}$ or PCBs. Comp. Biochem. Physiol. 118C: 11-18.

Landrum, P.F., Gedeon, M.L., Burton, G.A., Greenberg, M.S. \& RowLAND, C.D. 2002. Biological Responses of Lumbriculus variegatus Exposed to Fluoranthene-Spiked Sediment. Arch. Environ. Contam. Toxicol. 42: 292-302.

Laustein, G.G. 1995. Comparison of organic contaminants founding mussels and oysters from a current Mussel Watch Project with those from archived mollusk samples of the 1970s. Mar. Pollut. Bull. 30: 826-833.

Marrugo, A.J. 1993. Estudio de la contaminación marina por hidrocarburos en el Pacífico Colombiano - Fase III. Bol. Cient. CCCP. 4: 47-60.

Nair, D., Fernández-Acero, F.J., García-Luque, E., Riba, I. \& Del Valls, T.A. 2008. Isolation and characterization of naphthalene-degrading bacteria from sediments of Cadiz area (SW Spain). Environ. Toxicol. 23: 576-582.

Pane, L., Boccardo, S., Bonfiglioli, F., Mariottini, G.L., Priano, F. \& CONIO, O. 2005. Polycyclic aromatic hydrocarbons in water, seston and copepods in a harbor area in the Western Mediterranean (Ligurian Sea). Mar. Ecol. 26: 89-99.

Page, D.S., Boehm, P.D., Brown, J.S., Neff, J.M., Burns, W.A. \& Bence, A.E. 2005. Mussels document loss of bioavailable 
polycyclic aromatic hydrocarbons and the return to baseline conditions for oiled shorelines in Prince William Sound, Alaska. Mar. Environ. Res. 60, 422 -236.

Peña, A., Morales, J., Labastida, A. \& Capella, S. 2003. Extracción en Fase Sólida como una Alternativa para el Procedimiento de Limpieza en la Determinación de Hidrocarburos Aromáticos Policíclicos por Cromatografía de Gases. Rev. Int. Contam. Ambient. 19 (1): 13 - 23.

Prahl, H. von., Cantera, J.R., \& Contreras, R. 1990. Manglares y hombres del Pacífico colombiano. Fondo FEN. Bogotá, Colombia. 193 pp. p.

Russell, M.L., Goth-Goldstein, R., Apten, M.G. \& Fisk, W.J. 2002. Analytical Method for the determination of Individual (N-alkanes and Isoprenoids) and Total Petroleum Hydrocarbons (TPH), Polycyclic Aromatic Hydrocarbons
(PAHs) and Biomarker Triterpanes and Steranes in Oil and Oil-spill-related. Environmental Samples (Water, Particulates and Soils/Sediment): Berkeley (CA): Lawrence Berkeley National Laboratory, Environmental Energy Technologies Division; 2002 Jan. Report No.: LBNL49574. Contract No.: DEAC0376SF00098. Sponsored by the Department of Energy. Ottawa, ON, $\mathrm{K} 1 \mathrm{~A} 0 \mathrm{H} 3$.

Sericano, J.L., Wade, T.L., Jackson, T.J., Brooks, J. M., Tripp, B.W., FARRInGtON, J.W., Mee, L.D., Readman, J.W., Villeneuve, J.P. \& Goldbergs, E.D. 1995. Trace organic contamination in the Americas: An Overview of the US National Status \& Trends and the International "Mussel Watch" Programmes. Mar. Pollut. Bull. 31 (4-12): 214225.

Recibido: 13.12 .11

Aceptado: 29.02 .12 